\title{
Towards a Comprehensive TEM Toolbox for Complex Molecular Fluids
}

\author{
Min Gao
}

Liquid Crystal Institute, Kent State University, Kent, Ohio, USA.

In this paper, we summarize our ongoing efforts to set up a comprehensive yet easily accessible toolbox for TEM observation of complex molecular fluids (CMFs) and discuss the future possibilities that TEM community can develop for these fascinating but challenging materials. Many simple molecular fluids can be described using hard-sphere models, and have direct phase transitions from isotropic liquid phases to crystalline phases. Some nonspherical molecules can form complex structures, for example, micelles, or more interestingly, intermediate phases (the so-called liquid crystals, LCs) with orientational order only (nematic mesophases) or both orientational and 1D (or 2D in some cases) positional orders (smectic mesophases).

The CMFs have tremendous impacts (e.g., liquid crystal display or LCD, detergents, and crude oil) on our lives. However, the understanding of their detailed structures has been surprisingly limited due to their complicated structure and the lack of effective structural probe at subnanometer scale. For example, though initial direct TEM observation on the much studied CMF group - liquid crystals demonstrated promising results in late 1980's, the TEM studies were soon dominated by a replica TEM technique, namely freeze-fracture TEM (FFTEM). FFTEM effectively avoids two of the challenges for direct TEM observation on CMFs: i) difficulty in the preparation of thin TEM specimens to preserve the native structure partly due to the strong surface anchoring effect; ii) severe radiation damage. However, the relatively low resolution (a few nanometers) of FFTEM has hindered the understanding of CMF structures at the molecular scale.

In this study, a series of specimen preparation routines have been established for CMFs (Fig. 1), which have been applied to a variety of LC materials, including thermotropic (consisting of single- or multiplecomponent complex molecules) and lyotropic (complex molecules dissolved in certain solvents, for example, water) LCs [1]. Some new concepts include a simple procedure to produce suspended thermotropic LC thin films, combination of high-pressure freezing and cryo-ultramicrotomy for lyotropic LCs, and complementary applications of the direct cryo-TEM and FFTEM. We demonstrate that a widely available cryo-TEM, a high sensitivity CCD camera and a modified low-dose imaging procedure have made a ready combination for subnanometer resolution imaging of many challenging CMFs. We also explored the employment of diffraction, STEM Z-contrast, EDS, EELS and in situ observation of dynamic process. The significance of subnanometer resolution cryo-TEM observation is demonstrated in a few important issues in LC studies, including: i) revealing the existence of nanoscale layered smectic domains in nematic bent-core thermotropic LCs (Fig. 2) [1,2]; ii) providing evidence for the existence of twist-bend nematic phase with a complex director structure that follows the geometry of an oblique helicoid with a nanoscale pitch [1,3]; iii) probing the nematic packing of columnar aggregates in lyotropic chromonic liquid crystals (Fig. 2) [1,4]. More importantly, direct TEM observation opens ways to a variety of TEM techniques, suggesting that TEM (replica, cryo, and in situ techniques), in general, may be a promising part of the solution to the lack of effective structural probe at the molecular scale in CMF studies [5].

\section{References:}


[1] M Gao et al, Microsc Res Tech 77 (2014), 754.

[2] C Zhang et al, Phys Rev Lett 109 (2012), 107802.

[3] V Borshch et al, Nature Communications 4 (2013), 2635.

[4] D Pucci et al, J Mater Chem C 2 (2014), 8780.

[5] The TEM-related experiments were carried out at the cryo-TEM lab of the Liquid Crystal Institute, Kent State University. The author thanks Dr. Oleg D. Lavrentovich, Dr. Georg H. Mehl, and Dr. Wolfgang Weissflog for providing the samples.

(a)



(c)
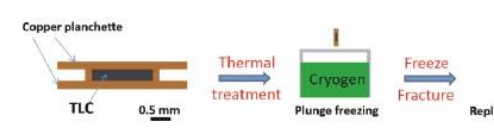
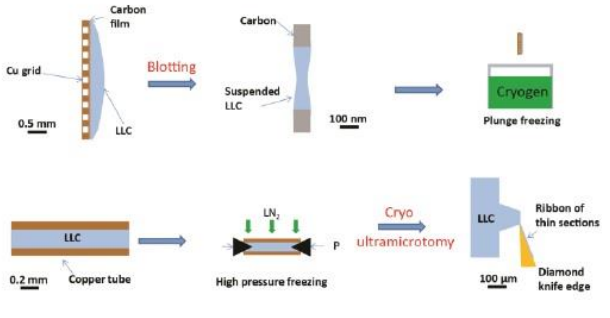

(f)

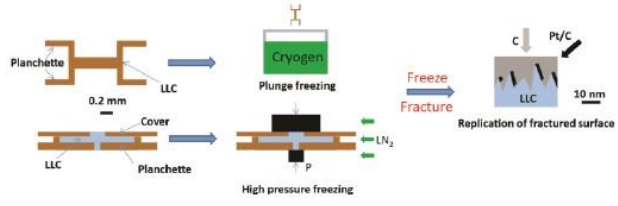

Figure 1. TEM specimen preparation routines for thermotropic liquid crystals (TLC) (a-c) and lyotropic liquid crystals (LLC) (d-f). (a) Plunge freezing of supported and suspended TLC thin films for cryoTEM. (b) Cryo-sectioning of plunge frozen "bulk" TLC for cryo-TEM. (c) Freeze fracture of plunge frozen "bulk" TLC for FFTEM. (d) Plunge freezing of LLC thin film for cryo-TEM. (e) Vitreous sectioning of high pressure frozen LLC for cryo-TEM. (f) Freeze fracture of frozen (plunge freezing or high pressure freezing) LLC for replica TEM. The scale bars in the schematics roughly demonstrate the feature sizes.



Figure 2. (a) Cryo-TEM image and corresponding FFT pattern showing layered smectic nanoclusters in a 3-ringe bent-core nematic liquid crystal (3RBC-N, the molecular structure is shown as an inset). The narrow solid arrows point out a few overlapping clusters, while the hollow arrows denote assemblies of side-by-side clusters. The lower insets are the magnified views of the $30 \mathrm{~nm} \times 30 \mathrm{~nm}$ junction areas marked by squares. An extra half layer is pointed out by a solid arrow. (b) - (d) Magnified images of representative complex assemblies of the layered smectic nanoclusters in 3RBC-N. The junctions are marked by hollow arrows, and the extra half layers by solid arrows. (e) - (f) Cryo-TEM images of the elongated aggregates in a silver(I) complex (20\% wt in water) with chromonic lyotropic LC behavior: side-view (e) and top-view (f). The insets in (e) are the molecule structure and the corresponding FFT. The inset in (f) shows a magnified image of the marked area $(30 \mathrm{~nm} \times 30 \mathrm{~nm})$ with a dashed square. 